\title{
A GEOMETRICAL CONFIGURATION WHICH IS A PARTIALLY BALANCED INCOMPLETE BLOCK DESIGN ${ }^{1}$
}

\author{
WILLARD H. CLATWORTHY ${ }^{2}$
}

1. Introduction. It will be shown that a geometrical configuration consisting of certain suitably chosen lines of the finite projective geometry $P G(3, s)$ may be interpreted as the partially balanced incomplete block design having parameters

$$
\begin{aligned}
v & =b=\left(s^{4}-1\right) /(s-1), \quad \lambda_{1}=1, \quad n_{1}=s(s+1), \\
r & =k=s+1, \quad \lambda_{2}=0, \quad n_{2}=s^{3}, \\
P_{1} & =\left(p_{j k}^{1}\right)=\left(\begin{array}{cc}
s-1 & s^{2} \\
s^{2} & s^{2}(s-1)
\end{array}\right), \\
P_{2} & =\left(p_{j k}^{2}\right)=\left(\begin{array}{cc}
s+1 & s^{2}-1 \\
s^{2}-1 & s^{2}(s-1)
\end{array}\right),
\end{aligned}
$$

where $s=p^{n}>1, p$ a prime and $n$ a positive integer. The design of (1.1) corresponding to $s=2$ was constructed by Bose and Shimamoto [4] by another method. All other designs of (1.1) are new.

Bose [1] and Bose and Nair [3] have used finite geometries and Galois fields in the construction of incomplete block designs. Once the geometrical configuration is determined, the proper assignment of the treatments to the blocks of design (1.1) may be determined by the geometrical method which is illustrated for the design corresponding to $s=3$.

2. A "working" definition of partially balanced incomplete block designs with two associate classes. Partially balanced incomplete block designs (P.B.I.B. designs) with $m$ associate classes $(m \geqq 1)$ were introduced by Bose and Nair [3]. Later Nair and Rao [5] broadened the definition of P.B.I.B. designs. Recently Bose and Shimamoto [4] rephrased the definition in order to stress the fact that the relations of association between the treatments are determined only by the parameters $n_{i}$ and $p_{j k}^{i}(i, j, k=1,2, \cdots, m)$. For the special case of P.B.I.B. designs with two associate classes $(m=2)$, Bose and Clatworthy [2] have proposed a new definition which to-

Received by the editors May 25, 1953.

1 This work was supported in part by The United States Air Force under Contract AF 18(600)-83 monitored by the Office of Scientific Research.

2 Now at Statistical Engineering Laboratory, National Bureau of Standards, Washington, D. C. 
gether with two theorems are equivalent to the Bose and Shimamoto definition. Use will be made in the present paper of the definition and theorems of [2] which are stated below.

Definition. A P.B.I.B. design with two associate classes is an arrangement of $v$ treatments in $b$ blocks such that:

(1) Each of the $v$ treatments occurs in $r$ blocks. Each block is of size $k$ (i.e., contains $k$ treatments), and no treatment appears more than once in any block.

(2) There exists a relationship of association between any pair of the $v$ treatments satisfying the following conditions:

(a) Any two treatments are either first or second associates.

(b) Each treatment has $n_{1}$ first and $n_{2}$ second associates.

(c) For any pair of the $v$ treatments which are $i$ th associates, the number of treatments common to the first associates of the first and the first associates of the second is $p_{11}^{i}(i=1,2)$, and this number is independent of the pair of treatments with which we start.

(3) Any pair of treatments which are $i$ th associates occur together in exactly $\lambda_{i}$ blocks $(i=1,2)$.

THEOREM 1. For every pair of first associates among the $v$ treatments of a P.B.I.B. design with two associate classes, the numbers $p_{12}^{1}, p_{21}^{1}$, and $p_{22}^{1}$ are constants, and $p_{12}^{1}=p_{21}^{1}$.

TheOREM 2. For every pair of second associates among the $v$ treatments of a P.B.I.B. design with two associate classes, the numbers $p_{12}^{2}$, $p_{21}^{2}$, and $p_{22}^{2}$ are constants, and $p_{12}^{2}=p_{21}^{2}$.

In proving Theorems 1 and 2 the following useful relations were established:

$$
\begin{aligned}
& p_{12}^{1}=p_{21}^{1}=n_{1}-p_{11}^{1}-1 \\
& p_{22}^{1}=n_{2}-n_{1}+p_{11}^{1}+1 \\
& p_{12}^{2}=p_{21}^{2}=n_{1}-p_{11}^{2} \\
& p_{22}^{2}=n_{2}-n_{1}+p_{11}^{2}-1 .
\end{aligned}
$$

The four preceding expressions are special cases of general expressions derived in [3] where the following relations were also established:

$$
\begin{aligned}
v r & =b k, \\
v & =n_{1}+n_{2}+1, \\
\lambda_{1} n_{1}+\lambda_{2} n_{2} & =r(k-1),
\end{aligned}
$$




$$
n_{1} p_{12}^{1}=n_{2} p_{11}^{2}, \quad n_{1} p_{22}^{1}=n_{2} p_{12}^{2}
$$

Furthermore, it was shown that if values are assigned to the parameters of the first kind $\left(v, b, r, k, \lambda_{1}, \lambda_{2}, n_{1}\right.$, and $\left.n_{2}\right)$ satisfying (2.5), (2.6), and (2.7), then only one of the parameters of the second kind $\left(p_{j k}^{i}, i, j, k=1,2\right)$ is independent.

The parameters of the second kind are exhibited as elements of two symmetric matrices,

$$
P_{1}=\left(\begin{array}{cc}
p_{11} & p_{12}^{1} \\
1 & 1 \\
p_{21} & p_{22}
\end{array}\right) \text { and } P_{2}=\left(\begin{array}{cc}
p_{11}^{2} & p_{12}^{2} \\
2 & 2 \\
p_{21} & p_{22}
\end{array}\right)
$$

3. The geometrical configuration. Consider the finite projective geometry $P G(3, s)$ which has $\left(s^{4}-1\right) /(s-1)$ points, $\left(s^{2}+1\right)\left(s^{2}+s+1\right)$ lines, and $\left(s^{4}-1\right) /(s-1)$ planes $[1]$. In this geometry the number of points contained in a line is $s+1$ and the number of points in a plane is $s^{2}+s+1$. Through any point there pass $s^{2}+s+1$ lines and the same number of planes. One line and $s+1$ planes pass through every pair of points in $P G(3, s)$.

The $\left(s^{4}-1\right) /(s-1)$ planes of $P G(3, s)$ in which we have an interest are chosen such that they are in (1-1) correspondence with the points of $P G(3, s)$. Let

$$
U_{i}=\sum_{j=1}^{4} a_{i j} x_{j} \quad(i=1,2,3,4)
$$

where $a_{i j}$ are elements of the Galois field $G F(s), s=p^{n}$, and the matrix $\left(a_{i j}\right)$ is skew-symmetric. To the point $\theta=\left(l_{1}, l_{2}, l_{3}, l_{4}\right)$ of $P G(3, s)$ let there correspond the plane $\pi_{\theta}$ whose equation is

$$
\sum_{i=1}^{4} l_{i} U_{i}=0
$$

We shall now establish the following

Lemma 3.1. (i) The plane $\pi_{\theta}$ passes through the point $\theta$.

(ii) If the plane $\pi_{\theta}$ passes through the point $\phi$, then the plane $\pi_{\phi}$ passes through point $\theta$.

By (3.1) and (3.2) the equation of the plane $\pi_{\theta}$ corresponding to point $\theta=\left(l_{1}, l_{2}, l_{3}, l_{4}\right)$ can be expressed in the form

$$
\sum_{i, j=1}^{4} a_{i j} l_{i} x_{j}=0
$$


Using the fact that the matrix $\left(a_{i j}\right)$ is skew-symmetric, (3.3) may be written as

$$
\begin{aligned}
& a_{12}\left(l_{1} x_{2}-l_{2} x_{1}\right)+a_{13}\left(l_{1} x_{3}-l_{3} x_{1}\right)+a_{14}\left(l_{1} x_{4}-l_{4} x_{1}\right) \\
& \quad+a_{23}\left(l_{2} x_{3}-l_{3} x_{2}\right)+a_{24}\left(l_{2} x_{4}-l_{4} x_{2}\right)+a_{34}\left(l_{3} x_{4}-l_{4} x_{3}\right)=0 .
\end{aligned}
$$

Obviously equation (3.4) is satisfied when

$$
\left(x_{1}, x_{2}, x_{3}, x_{4}\right)=\left(l_{1}, l_{2}, l_{3}, l_{4}\right)=\theta .
$$

Hence point $\theta$ lies in plane $\pi_{\theta}$.

Let point $\phi$ be denoted by $\left(m_{1}, m_{2}, m_{3}, m_{4}\right)$. Since plane $\pi_{\theta}$ corresponding to point $\theta$ passes through point $\phi$ by hypothesis, we have from (3.3)

$$
\sum_{i, j=1}^{4} a_{i j} l_{i} m_{j}=0
$$

The plane $\pi_{\phi}$ corresponding to point $\phi$ is

$$
\sum_{i=1}^{4} m_{i} U_{i}=0
$$

or

$$
\sum_{i, j=1}^{4} a_{i j} m_{i} x_{j}=0 .
$$

For plane $\pi_{\phi}$ to pass through point $\theta$ it is necessary that the coordinates of $\theta$ satisfy (3.8), i.e.,

$$
\sum_{i, j=1}^{4} a_{i j} m_{i} l_{j}=0
$$

Since matrix $\left(a_{i j}\right)$ is skew-symmetric, (3.9) becomes

$$
-\sum_{i, j=1}^{4} a_{j i} m_{i} l_{j}=0
$$

which is identical to (3.6). Therefore, if plane $\pi_{\theta}$ passes through point $\phi$, then plane $\pi_{\phi}$ passes through point $\theta$.

4. Interpretation of the geometrical configuration as design (1.1). We now identify the $\left(s^{4}-1\right) /(s-1)$ points of $P G(3, s)$ with the $\left(s^{4}-1\right) /(s-1)$ treatments of design (1.1) such that a point of $P G(3, s)$ corresponds to one and only one treatment of the design.

First, we establish the relation of association for design (1.1). We shall say that the point (or treatment) $\phi$ is a first associate of point 
$\theta$ if the plane $\pi_{\theta}$ corresponding to $\theta$ passes through point $\phi$. Let $\phi$ be a first associate of $\theta$. Then by Lemma 3.1, plane $\pi_{\phi}$ passes through point $\theta$; hence, point $\theta$ is by definition a first associate of point $\phi$. Thus the association relation is reflexive as it should be. By definition, any point $\alpha$ which is not a first associate of $\theta$ is its second associate.

We shall now prove that condition (c) of (2) of the definition of a P.B.I.B. design with two associate classes is satisfied.

(i) Since there are $s^{2}+s$ points lying in $\pi_{\theta}$ other than $\theta$ itself, the number of first associates of $\theta$ is

$$
n_{1}=s^{2}+s .
$$

Since the total number of points in $P G(3, s)$ is $s^{3}+s^{2}+s+1$, the number of second associates of $\theta$ is

$$
n_{2}=s^{3} \text {. }
$$

(ii) Let points $\theta$ and $\phi$ be first associates. Points which are first associates of both $\theta$ and $\phi$ must lie in the line of intersection of planes $\pi_{\theta}$ and $\pi_{\phi}$ passing through $\theta$ and $\phi$ respectively. This line passes through both $\theta$ and $\phi$. It contains $s-1$ points other than $\theta$ and $\phi$. Hence the number of points common to the first associates of $\theta$ and $\phi$ is

$$
p_{11}^{1}=s-1
$$

Next, let points $\theta$ and $\phi$ be second associates. The points which are first associates of both $\theta$ and $\phi$ must lie on the line of intersection of the planes $\pi_{\theta}$ and $\pi_{\phi}$ as before. However, this line no longer passes through points $\theta$ and $\phi$. Since a line contains $s+1$ points, the number of treatments common to the first associates of $\theta$ and $\phi$ is

$$
p_{11}^{2}=s+1 \text {. }
$$

It now follows from Theorems 1 and 2 and expressions (2.1) through (2.4) that

$$
\begin{aligned}
& p_{12}^{1}=p_{21}^{1}=n_{1}-p_{11}^{1}-1=s^{2}, \\
& p_{22}^{1}=n_{2}-n_{1}+p_{11}^{1}+1=s^{2}(s-1), \\
& p_{12}^{2}=p_{21}^{2}=n_{1}-p_{11}^{2}=s^{2}-1, \\
& p_{22}^{2}=n_{2}-n_{1}+p_{11}^{2}-1=s^{2}(s-1) .
\end{aligned}
$$

It remains to show that the conditions on parameters $b, r, k, \lambda_{1}$, and $\lambda_{2}$ of design (1.1) are satisfied. Let $l_{\theta}$ be any line passing through 
$\theta$ and lying in the plane $\pi_{\theta}$. The set of all points contained in line $l_{\theta}$ is taken to form one block of our design. Since there are $s+1$ lines passing through $\theta$ and lying in $\pi_{\theta}$, there are $s+1$ blocks containing treatment $\theta$. Hence

$$
r=k=s+1 \text {. }
$$

The total number of points in $P G(3, s)$ is $\left(s^{4}-1\right) /(s-1)$. Hence the total number of lines like $l_{\theta}$ is $\left(s^{4}-1\right) /(s-1)$ since through each point there pass $s+1$ lines like $l_{\theta}$, and on each line there lie $s+1$ points. Thus the number of blocks is

$$
b=\left(s^{4}-1\right) /(s-1) .
$$

If $\theta$ and $\phi$ are first associates, then the line joining $\theta$ and $\phi$ is a block. Hence any two first associates occur together in exactly one block, giving

$$
\lambda_{1}=1 \text {. }
$$

If $\theta$ and $\phi$ are second associates, neither of the corresponding planes $\pi_{\theta}$ and $\pi_{\phi}$ contains both $\theta$ and $\phi$. Hence the lines $l_{\theta}$ passing through $\theta$ never pass through $\phi$ and, therefore, the blocks containing $\theta$ cannot contain $\phi$. Thus

$$
\lambda_{2}=0 .
$$

This completes the proof that the system of blocks defined above satisfies design (1.1).

5. Construction of the design with $s=3$. The geometrical method of construction of design (1.1) will be illustrated by considering the design corresponding to $s=3$. This design has parameters

$$
\begin{aligned}
& v=b=40, \lambda_{1}=1, n_{1}=12, P_{1}=\left(\begin{array}{lr}
2 & 9 \\
9 & 18
\end{array}\right), \\
& r=k=4, \quad \lambda_{2}=0, n_{2}=27, P_{2}=\left(\begin{array}{rr}
4 & 8 \\
8 & 18
\end{array}\right) .
\end{aligned}
$$

We use $P G(3,3)$ and the Galois field $G F(3)$ whose $s=3$ elements are 0,1 , and 2 . Let the skew-symmetric matrix be

$$
\left(a_{i j}\right)=\left(\begin{array}{ccccc}
0 & 1 & : & & \\
2 & 0 & : & & 0 \\
\cdot & \cdot & : & \cdot & \cdot \\
0 & \vdots & 0 & 2 \\
& & \cdot & 1 & 0
\end{array}\right) .
$$


The plane of $P G(3,3)$ corresponding to point $\theta=\left(l_{1}, l_{2}, l_{3}, l_{4}\right)$ is

$$
l_{1} x_{2}+2 l_{2} x_{1}+2 l_{3} x_{4}+l_{4} x_{3}=0 .
$$

In order to have a one-to-one correspondence between sets of coordinates with elements in $G F(3)$ and the 40 points of $P G(3,3)$, we shall restrict ourselves to the set of coordinates in which the left-most nonzero digit is unity. In order to express the blocks of the design in terms familiar to the experimentalist, we set up a correspondence between the points of $P G(3,3)$ and the integers $1,2,3, \ldots, 40$ according to the following rule: Let $\left(l_{1}, l_{2}, l_{3}, l_{4}\right)$ correspond to

$$
l_{1} s^{3}+l_{2} s^{2}+l_{3} s+l_{4}-\alpha
$$

where the number $\alpha$ is defined by

$$
\begin{aligned}
& \alpha=s^{2}+s+1 \\
& \alpha=s+1 \\
& \alpha=1 \\
& \alpha=0
\end{aligned}
$$$$
\text { if } l_{1}=1 \text {, }
$$$$
\text { if } l_{1}=0 \text { and } l_{2}=1 \text {, }
$$$$
\text { if } l_{1}=l_{2}=0 \text { and } l_{3}=1 \text {, }
$$$$
\text { if } l_{1}=l_{2}=l_{3}=0 \text { and } l_{4}=1 \text {. }
$$

The solution of design (5.1) is exhibited below in tabular form. It will be noted that only 13 of the 40 planes are exhibited in the solution. The points of the other 27 planes lie in the 13 planes indicated in column (2).

6. Acknowledgement. The writer is indebted to Dr. R. C. Bose for his guidance in this paper.

SOLuTION OF DESIGN (5.1)

\begin{tabular}{ccccccccccc}
\hline \hline $\begin{array}{c}\text { (1) } \\
\text { Point }\end{array}$ & $\begin{array}{c}(2) \\
\text { Plane Corresp. } \\
\text { to Point }\end{array}$ & \multicolumn{3}{c}{$\begin{array}{c}\text { (3) } \\
\text { Points Lying in Lines } \\
\text { (blocks) of Plane }\end{array}$} & \multicolumn{5}{c}{ Blocks of (5.1) } \\
\hline 0001 & $x_{3}=0$ & 0001 & 0100 & 0101 & 0102 & 1 & 5 & 6 & 7 \\
& & 0001 & 1000 & 1001 & 1002 & 1 & 14 & 15 & 16 \\
& & 0001 & 1100 & 1101 & 1102 & 1 & 23 & 24 & 25 \\
& & 0001 & 1200 & 1201 & 1202 & 1 & 32 & 33 & 34 \\
\hline 0100 & \multirow{2}{*}{$x_{1}=0$} & 0100 & 0010 & 0110 & 0120 & 5 & 2 & 8 & 11 \\
& & 0100 & 0011 & 0111 & 0122 & 5 & 3 & 9 & 13 \\
& & 0100 & 0012 & 0112 & 0121 & 5 & 4 & 10 & 12 \\
\hline 0101 & $2 x_{1}+x_{3}=0$ & 0101 & 1010 & 1111 & 1212 & 6 & 17 & 27 & 37 \\
& & 0101 & 1011 & 1112 & 1210 & 6 & 18 & 28 & 35 \\
& & 0101 & 1012 & 1110 & 1211 & 6 & 19 & 26 & 36 \\
\hline
\end{tabular}


SLUTIOON OF DESIGN (5.1) (continued)

(1) (2)

Point Plane Corresp. to Point
(3)

Points Lying in Lines

(blocks) of Plane
(4)

Blocks of (5.1)

\begin{tabular}{|c|c|c|c|c|c|c|c|c|c|}
\hline 0102 & $x_{1}+x_{3}=0$ & $\begin{array}{l}0102 \\
0102 \\
0102\end{array}$ & $\begin{array}{l}1020 \\
1021 \\
1022\end{array}$ & $\begin{array}{l}1122 \\
1120 \\
1121\end{array}$ & $\begin{array}{l}1221 \\
1222 \\
1220\end{array}$ & $\begin{array}{l}7 \\
7 \\
7\end{array}$ & $\begin{array}{l}20 \\
21 \\
22\end{array}$ & $\begin{array}{l}31 \\
29 \\
30\end{array}$ & $\begin{array}{l}39 \\
40 \\
38\end{array}$ \\
\hline \multirow[t]{3}{*}{1000} & $x_{2}=0$ & 1000 & 0010 & 1010 & 1020 & 14 & 2 & 17 & 20 \\
\hline & & 1000 & 0011 & 1011 & 1022 & 14 & 3 & 18 & 22 \\
\hline & & 1000 & 0012 & 1012 & 1021 & 14 & 4 & 19 & 21 \\
\hline \multirow[t]{3}{*}{1001} & $x_{2}+x_{3}=0$ & 1001 & 0120 & 1121 & 1211 & 15 & 11 & 30 & 36 \\
\hline & & 1001 & 0121 & 1122 & 1210 & 15 & 12 & 31 & 35 \\
\hline & & 1001 & 0122 & 1120 & 1212 & 15 & 13 & 29 & 37 \\
\hline \multirow[t]{3}{*}{1002} & $x_{2}+2 x_{3}=0$ & 1002 & 0110 & 1112 & 1222 & 16 & 8 & 28 & 40 \\
\hline & & 1002 & 0111 & 1110 & 1221 & 16 & 9 & 26 & 39 \\
\hline & & 1002 & 0112 & 1111 & 1220 & 16 & 10 & 27 & 38 \\
\hline \multirow[t]{3}{*}{1100} & $x_{2}+2 x_{1}=0$ & 1100 & 0010 & 1110 & 1120 & 23 & 2 & 26 & 29 \\
\hline & & 1100 & 0011 & 1111 & 1122 & 23 & 3 & 27 & 31 \\
\hline & & 1100 & 0012 & 1112 & 1121 & 23 & 4 & 28 & 30 \\
\hline \multirow[t]{3}{*}{1101} & $2 x_{1}+x_{2}+x_{3}=0$ & 1101 & 0120 & 1011 & 1221 & 24 & 11 & 18 & 39 \\
\hline & & 1101 & 0121 & 1010 & 1222 & 24 & 12 & 17 & 40 \\
\hline & & 1101 & 0122 & 1012 & 1220 & 24 & 13 & 19 & 38 \\
\hline \multirow[t]{3}{*}{1102} & $2 x_{1}+x_{2}+2 x_{3}=0$ & 1102 & 0110 & 1022 & 1212 & 25 & 8 & 22 & 37 \\
\hline & & 1102 & 0111 & 1021 & 1210 & 25 & 9 & 21 & 35 \\
\hline & & 1102 & 0112 & 1020 & 1211 & 25 & 10 & 20 & 36 \\
\hline \multirow[t]{3}{*}{1200} & $x_{1}+x_{2}=0$ & 1200 & 0010 & 1210 & 1220 & 32 & 2 & 35 & 38 \\
\hline & & 1200 & 0011 & 1211 & 1222 & 32 & 3 & 36 & 40 \\
\hline & & 1200 & 0012 & 1212 & 1221 & 32 & 4 & 37 & 39 \\
\hline \multirow[t]{3}{*}{1201} & $x_{1}+x_{2}+x_{3}=0$ & 1201 & 0120 & 1021 & 1111 & 33 & 11 & 21 & 27 \\
\hline & & 1201 & 0121 & 1022 & 1110 & 33 & 12 & 22 & 26 \\
\hline & & 1201 & 0122 & 1020 & 1112 & 33 & 13 & 20 & 28 \\
\hline \multirow[t]{3}{*}{1202} & $x_{1}+x_{2}+2 x_{3}=0$ & 1202 & 0110 & 1012 & 1122 & 34 & 8 & 19 & 31 \\
\hline & & 1202 & 0111 & 1010 & 1121 & 34 & 9 & 17 & 30 \\
\hline & & 1202 & 0112 & 1011 & 1120 & 34 & 10 & 18 & 29 \\
\hline
\end{tabular}

\section{REFERENCES}

1. R. C. Bose, On the construction of balanced incomplete block designs, Annals of Eugenics vol. 9 (1939) pp. 353-399. 
2. R. C. Bose and W. H. Clatworthy, Partially balanced designs with two associate classes and $k>r=3, \lambda_{1}=1, \lambda_{2}=0$, in preparation for publication.

3. R. C. Bose and K. R. Nair, Partially balanced incomplete block designs, Sankhyā vol. 4 (1939) pp. 337-372.

4. R. C. Bose and T. Shimamoto, Classification and analysis of partially balanced incomplete block designs with two associate classes, Journal of the American Statistical Association vol. 47 (1952) pp. 151-184.

5. K. R. Nair and C. R. Rao, A note on partially balanced incomplete block designs, Science and Culture vol. 7 (1942) pp. 568-569.

University of North Carolina

\section{SOME CONDITIONS FOR UNIFORM CONVERGENCE OF INTEGRALS}

\section{EMANUEL PARZEN ${ }^{1}$}

Let $m$ be a sigma-finite measure on a space $R$, let $T$ be an abstract index set, and for each $t \in T$ and $n=1,2, \cdots$, let $f_{n}(x, t), f(x, t)$ be functions measurable in $x \in R$. It is of some interest to determine conditions for statements of the form

$$
\int_{R}\left|f_{n}(x, t)-f(x, t)\right| d m \rightarrow 0 \quad \text { uniformly in } t,
$$

where we use the conventions that if a limit is taken as $n \rightarrow \infty$, or if $t$ varies in $T$, then these facts will not be explicitly stated. Graves $[1$, p. 241, Theorem 22] states conditions for (1) to hold under the assumption that:

(2) at each $x \in R$, except for $x$ in a null set which is independent of $t, f_{n}(x, t)$ converges to $f(x, t)$ uniformly in $t$.

The mode of convergence defined by (2) is much too restrictive. In this paper we define modes of uniform convergence of a family of sequences of measurable functions which are much less restrictive than (2) and under which (1) holds. Situations of this more general kind were encountered by the author in [2], and the theorems proved below are there applied.

Before introducing our modes of convergence, let us recall that, given measurable functions $f, f_{n}(n=1,2, \cdots)$, Egorov's theorem states that $f_{n}(x) \rightarrow f(x)$ almost everywhere (that is, $m\left\{x: f_{n}(x)\right.$ does not converge to $f(x)\}=0$ ) if, and only if, for every $\epsilon>0$ and every

Presented to the Society, September 4, 1953; received by the editors March 30, 1953 and, in revised form, June 1, 1953.

1 National Science Foundation predoctoral fellow, 1952-53. Now at Columbia University, Hudson Laboratories, Dobbs Ferry, New York. 